\title{
VALIDATION AND QUANTITATIVE ANALYSIS OF CARMINE AND RHODAMINE B IN LIPSTICK FORMULATION
}

\author{
REYNA NEVITASARI1,2, ABDUL ROHMAN ${ }^{1,3 *}{ }^{*}$, SUDIBYO MARTONO1
}

${ }^{1}$ Department of Pharmaceutical Chemistry, Faculty of Pharmacy, Universitas Gadjah Mada, Yogyakarta, ${ }^{2}$ The National Agency of Drug and Food Control, District of Surabaya, East Java Republic of Indonesia, ${ }^{3}$ Institute of Halal Industry and System , Universitas Gadjah Mada, Yogyakarta.

Email: abdul_kimfar@ugm.ac.id

Received: 16 Feb 2019, Revised and Accepted: 06 Apr 2019

\section{ABSTRACT}

Objective: The objective of this study was to validate reversed phase-high performance liquid chromatography (RP-HPLC) method using photodiode array detector for the simultaneous determination of carmine (CAR) and rhodamine B (RHO B) in lipstick products.

Methods: CAR and RHO B were analysed using Cosmosil C18 column (250 mm x $4.6 \mathrm{~mm}$ i.d., $5 \mu \mathrm{m}$ ) using Shimadzu LC 20AD chromatograph equipped with photo-diode array (PDA) detector at 245-600 nm. The mobile phase used was acetonitrile-phosphate buffer pH 3.4 (5.5:4.5 v/v) delivered isocratically at flow rate of $1.0 \mathrm{ml} / \mathrm{min}$, using column temperature at $35^{\circ} \mathrm{C}$. The optimized HPLC condition was validated by determining several characteristics including selectivity, linearity, sensitivity expressed by the limit of detection and limit of quantification, precision and accuracy.

Results: The optimized HPLC condition could separate CAR and RHO B successfully with resolution value>2.0. The method was linear over studied concentration with a coefficient of determination $\left(R^{2}\right) \geq 0.9999$. The relative standard deviation (RSD) values were lower than those required RSD Horwitz and the recovery values were also acceptable, therefore, the developed method was precise and accurate.

Conclusion: HPLC with PDA detector has been accurately and successfully used for quantitative analysis of CAR and RHO B. The developed method is simple and can be used for routine analysis of CAR and RHO B for quality assurance purposes.

Keywords: Carmine, Rhodamine B, RP-HPLC-PDA, Validation, Lipstick products

(C) 2019 The Authors. Published by Innovare Academic Sciences Pvt Ltd. This is an open-access article under the CC BY license (http://creativecommons.org/licenses/by/4.0/) DOI: http://dx.doi.org/10.22159/ijap.2019v11i3.32492

\section{INTRODUCTION}

Cosmetic products are widely used especially for woman and are directly applied to human skin. One of the cosmetics products commonly used is lipstick and eye shadow [1]. Lipstick use has been hypothesized to be a risk factor of developing systemic lupus erythematosus in human [2], therefore the assessment of lipstick safety through quantitative analysis of components present in lipstick is necessary. Dyes are the most important additive component in the cosmetics industry to improve personal appearance [3]. Carmine (CAR) and Rhodamine B (RHO B) are among dyes used in lipstick formulation. The chemical structures of CAR and RHO B are depicted in fig. 1. CAR, natural colorant, is allowed in cosmetics product, while RHO B, a synthetic dye, is not allowed to be used in cosmetics products. Due to its similarity colour, CAR is frequently replaced by RHO B $[4,5]$.

CAR is one of the natural dyes relatively safe to be used in food industry especially in yogurt as well as in cosmetics products of lipstick and eye shadow. Allergenic reaction (shock anaphylaxis) has been reported due to food consumption containing CAR [6]. In addition, RHO B is one of the synthetic dyes banned to be used in food and cosmetics products. The exposure of RHO B in food and cosmetics for long time has been associated with cancer and liver diseases [7]. Therefore, analytical methods for quantitative analysis of CAR and RHO B must be developed in order to ensure the cosmetic safety.

Several publications have reported determination of CAR and RHO B in cosmetics and food products either individually or simultaneously. Such methods are high-performance liquid chromatography with photo-diode array detector [8, 9], liquid chromatography-mass spectrometry (LC-MS) tandem with MS using electrospray ionization [10], magnetic molecularly imprinted polymer characterized by scanning electron microscopy, FTIR spectroscopy and thermo-gravimetric analysis [11], and Fourier transform infrared spectroscopy using attenuated total reflectance. In this study, HPLC with PDA detector using experimental design approach has been optimized and validated for quantitative analysis of CAR and RHO B in lipstick formulation.<smiles>Cc1c(C(=O)O)c(O)cc2c1C(=O)C1C(O)=C(C3=C(O)[C@@H](O)[C@H](O)C(CO)O3)C(O)=C(O)C1C2=O</smiles>

Carmine<smiles>CCN(CC)C1=CC2OC3=CC(=[N+](C)C)C=CC3C(c3ccccc3C(=O)O)=C2C=C1</smiles>

Rhodamine B

Fig. 1: The chemical structures of Carmine (CAR) and Rhodamine B (RHO B)

\section{MATERIALS AND METHODS}

Lipstick products were obtained from several local markets and supermarkets around Yogyakarta. The reference standards of Carmine (CAR) and Rhodamine B (RHO B) were acquired from the 
national agency of drug and food control (NADFC) of Republic of Indonesia. All solvents used for mobile phase were of HPLC grade and obtained from E. Merck (Darmstadt, Germany). Aquabidest was obtained from Ikapharmindo (Indonesia).

\section{Preparation of reference standards}

For preparation of stock solution, an approximately of $5.00 \mathrm{mg}$ of each CAR and RHO B was accurately weighed using analytical balance (Metler Toledo MX5) with sensitivity of $0.01 \mathrm{mg}$ and was added into volumetric flask $50 \mathrm{ml}$ and dissolved with mobile phase until volume. This solution was then used for preparing calibration curve.

\section{Preparation of samples}

An approximately of $1000.0 \mathrm{mg}$ of lipstick products was accurately weighed using analytical balance (Metler Toledo MX5) with sensitivity of $0.1 \mathrm{mg}$, and dissolved with mobile phase until $10.0 \mathrm{ml}$. The solution was filtered with PTFE $0.45 \mu \mathrm{m}$ and injected into HPLC system.

\section{HPLC instrumentation}

CAR and RHO B was separated by Cosmosil C18 column $(250 \mathrm{~mm} x$ $4.6 \mathrm{~mm}$ i.d.,5 $\mu \mathrm{m}$ ) using Shimadzu LC 20AD chromatograph equipped with photo-diode array (PDA) detector at 245-600 nm. The mobile phase used was acetonitrile-phosphate buffer $\mathrm{pH} 3.4$ $(5.5: 4.5 \mathrm{v} / \mathrm{v})$ delivered isocratically at flow rate of $1.0 \mathrm{ml} / \mathrm{min}$, using column temperature at $35^{\circ} \mathrm{C}$.

\section{Validation of analytical method}

Validation of analytical method is needed to assure that the method fits to its purposes [13]. HPLC using optimum condition was validated by assessing several performance characteristics including selectivity, linearity, sensitivity expressed by limit of detection and limit of quantification, precision and accuracy as in International Conference on Harmonization (ICH) guidelines 2015 [14,15].

\section{Data analysis}

All validation data (linear regression, recovery, relative standard deviation (RSD) and recovery) were expressed as mean \pm standard deviation (SD) of mean and were calculated using Microsoft Excel (Microsoft Inc., USA).

\section{RESULTS AND DISCUSSION}

Reversed phase high-performance liquid chromatography (RPHPLC) is the most commonly methods used for separation of dyes including CAR and RHO B because of its accuracy, precision, sensitivity, time efficiency, low cost and robust [16]. The different polarity between CAR and RHO B could be separated using C18 column. Fig. 2 exhibited the separation of CAR and RHO B using optimized condition. Both analytes (CAR and RHO B) were well separated with resolution value (Rs) of 15.10 indicating that RPHPLC was selective. The system suitability test of RP-HPLC indicated that RP-HPLC system was reliable for quantitative analysis of CAR and $\mathrm{RHO} \mathrm{B}$ as indicated that RSD values of retention times of CAR and RHO B as well as peak areas of CAR and RHO B were less than $2 \%[17]$.

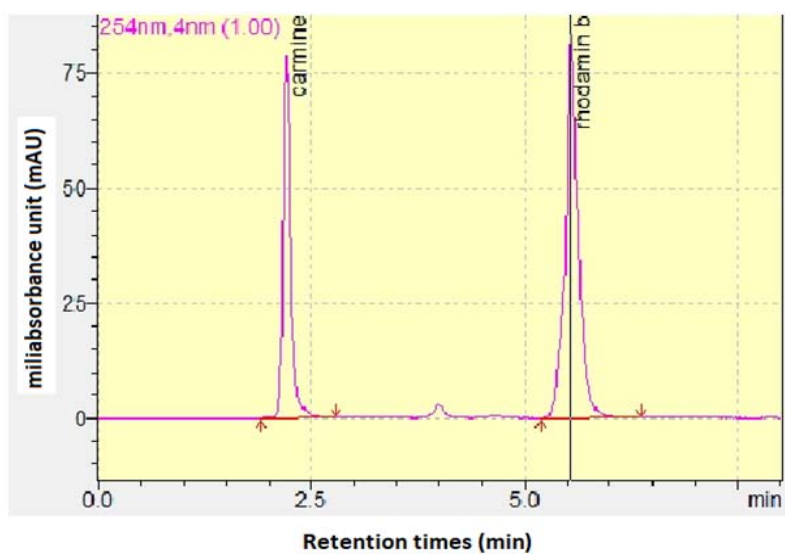

Fig. 2: The separation of Carmine and Rhodamine B using the optimized RP-HPLC condition

The advantage of PDA detector is the capability to provide the purity index of spectra of corresponding analytes (CAR and RHO B). PDA detector can analyze peak purity by comparing spectra of analytes within a peak. A pure peak has matching spectra throughout the peak (at all wavelengths). Both CAR and RHO B have the purity index $>0.9999$ and were considered as pure, meaning that the eluted peaks were of CAR and RHO B. PDA detector can also monitor analytes at more than one wavelength. This is especially useful when the wavelength maxima of the analytes are different. Wavelengths can be selected to analyse each compound at its highest sensitivity. Fig. 3 revealed the 3D spectra of CAR and RHO B at wavelengths of $245-600 \mathrm{~nm}$.

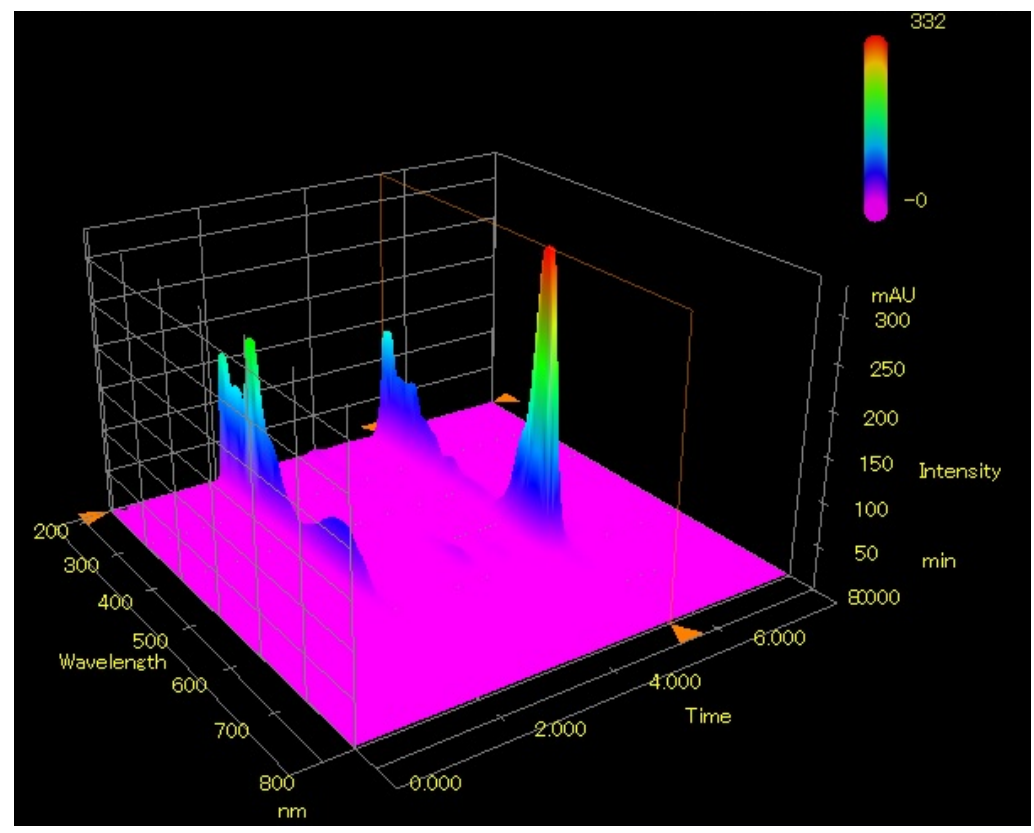

Fig. 3: The 3D spectra of carmine (left) and Rhodamine B (right) at wavelengths of 245-600 nm 

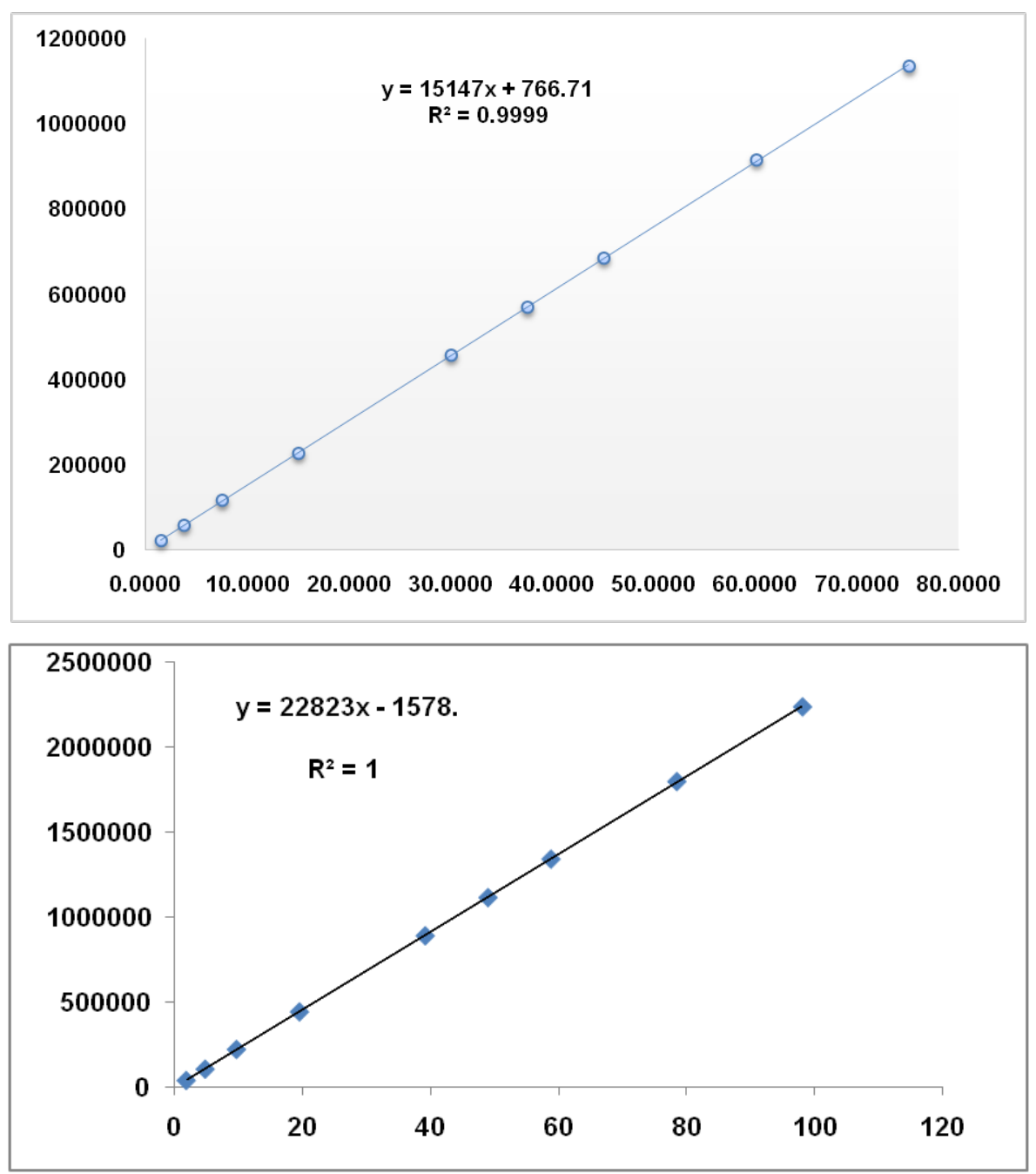

Fig. 4: The relationship between the concentration of carmine and rhodamine $B(x-a x i s)$ and peak area (y-axis)

RP-HPLC method was validated according to ICH (2015) guideline by evaluating some performance characteristics which include linearity and range, sensitivity as expressed by LoD and LoQ, precision expressed as RSD of repeatability and intermediate precision and accuracy expressed by recovery of analytes added in the lipstick samples. Linearity of RP-HPLC was evaluated by preparing a series of standard solutions of CAR and RHO B with certain concentration and correlating between the concentrations of both analytes (x-axis) and peak area (y-axis). Fig. 4 revealed the equation of linear regression describing the correlation between concentration and peak area as indicated by the coefficient of determination $\left(\mathrm{R}^{2}\right)$. The $\mathrm{R}^{2}$-values were higher than 0.999 which indicated that linear relationship existed between concentration and peak area at certain concentration. In addition, \%-intercept values were also low $(<2 \%)$ indicating the absence of systematic error.

The sensitivity of RP-HPLC with PDA detector for quantitative analysis of CAR and RHO B was expressed by values of limit of detection (LoD) and limit of quantification. The values of LoD obtained were $6.42 \mathrm{ng} / \mathrm{ml}$ and $3.85 \mathrm{ng} / \mathrm{ml}$ LoQ for CAR and RHO B, respectively. In addition LoQ values were calculated as: $3.33 \times \mathrm{LoD}$ values. The LoQ values obtained were $21.78 \mathrm{ng} / \mathrm{ml}$ and $12.82 \mathrm{ng} / \mathrm{ml}$ for CAR and RHO B, respectively.

The precision of RP-HPLC using PDA detector was assessed by computing values of relative standard deviation (RSD) during repeatability (intra-assay) and intermediate precision (inter-assay) using different days of analysis. The RP-HPLC method was considered precise if RSD values obtained were lower than those in
Horwitz criteria $[17,18]$. The RSD values of CAR and RHO B during repeatability at concentration levels of $24 \mu \mathrm{g} / \mathrm{ml}$ with six replicates $(\mathrm{n}=6)$ were $1.12 \%$ and $1.60 \%$, respectively. In addition, RSD values for intermediate precision were of $1.25 \%$ and $1.22 \%$ for CAR and RHO B, respectively. The maximum RSD Horwitz for analytes with level of $24.0 \mu \mathrm{g} / \mathrm{ml}$ was $7.3 \%$, therefore, all RSD values during repeatability (intra-assay) and intermediate precision (inter-assay) were lower than 7.3\% [18], therefore, RP-HPLC was precise enough for quantitative analysis of CAR and RHO B in lipstick products.

The accuracy of RP-HPLC was evaluated by recovery percentage using standard addition method. In this case, reference standard solutions with known concentrations of CAR and RHO B at three different concentrations (i.e. $80 \%, 100 \%$, and $120 \%$ from target analytes) were added into blank lipstick samples (no detectable analytes). Table 1 and 2 showed the recovery percentages of CAR and RHO B as analysed using RP-HPLC. The Association of Official Analytical Chemists (AOAC) set up that the recovery percentage of analytes at level $<100 \mathrm{ppm}$ $(\mu \mathrm{g} / \mathrm{g})$ was in the range of $80-110 \%$ [18], therefore, the recovery percentages of CAR and RHO B obtained using RP-HPLC was acceptable, meaning that this method was accurate. It was also stated that systematic errors could be negligible [19].

Based on validation performance characteristics evaluated, it can be concluded that RP-HPLC with PDA detector at wavelength of $254 \mathrm{~nm}$ was valid for quantitative analysis of CAR and RHO B in lipstick samples. The validated RP-HPLC method was further used for quantitative analysis of CAR and RHO B. Fig. 5 revealed RP-HPLC chromatogram of commercial lipstick. All lipstick samples analysed were not detected to contain CAR and RHO B. 
Table 1: The recovery percentages of Carmine as analysed using reversed phase-high performance liquid chromatography

\begin{tabular}{|c|c|c|c|}
\hline $\begin{array}{l}\text { The levels of standard spiked into lipstick } \\
(\mu \mathrm{g} / \mathrm{g})\end{array}$ & The concentration found $(\mu \mathrm{g} / \mathrm{g})$ & The percentage recovery & $\begin{array}{l}\text { Acceptance criteria } \\
(\%)\end{array}$ \\
\hline 9.88 & 7.91 & 80.05 & $80-110$ \\
\hline 9.88 & 7.96 & 80.55 & $80-110$ \\
\hline 9.88 & 8.536 & 86.37 & $80-110$ \\
\hline 30.09 & 24.56 & 81.62 & $80-110$ \\
\hline 30.09 & 25.91 & 86.12 & $80-110$ \\
\hline 30.09 & 26.66 & 88.60 & $80-110$ \\
\hline 49.88 & 40.17 & 80.54 & $80-110$ \\
\hline 49.88 & 46.25 & 92.72 & $80-110$ \\
\hline 49.88 & 45.61 & 91.44 & $80-110$ \\
\hline
\end{tabular}

Table 2: The recovery percentages of Rhodamine B as analysed using reversed phase-high performance liquid chromatography

\begin{tabular}{llll}
\hline The levels of standard spiked into lipstick (mg) & The concentration found (mg) & The percentage recovery & Acceptance criteria (\%) \\
\hline 9.88 & 9.53 & 96.43 & $80-110$ \\
9.88 & 9.41 & 95.26 & $80-110$ \\
9.88 & 10.74 & 108.74 & $80-110$ \\
30.09 & 32.42 & 107.75 & $80-110$ \\
30.09 & 32.52 & 108.06 & $80-110$ \\
30.09 & 32.06 & 106.55 & $80-110$ \\
49.88 & 52.84 & 105.94 & $80-110$ \\
49.88 & 46.28 & 92.78 & $80-110$ \\
49.88 & 44.30 & 88.82 & $80-110$ \\
\hline
\end{tabular}
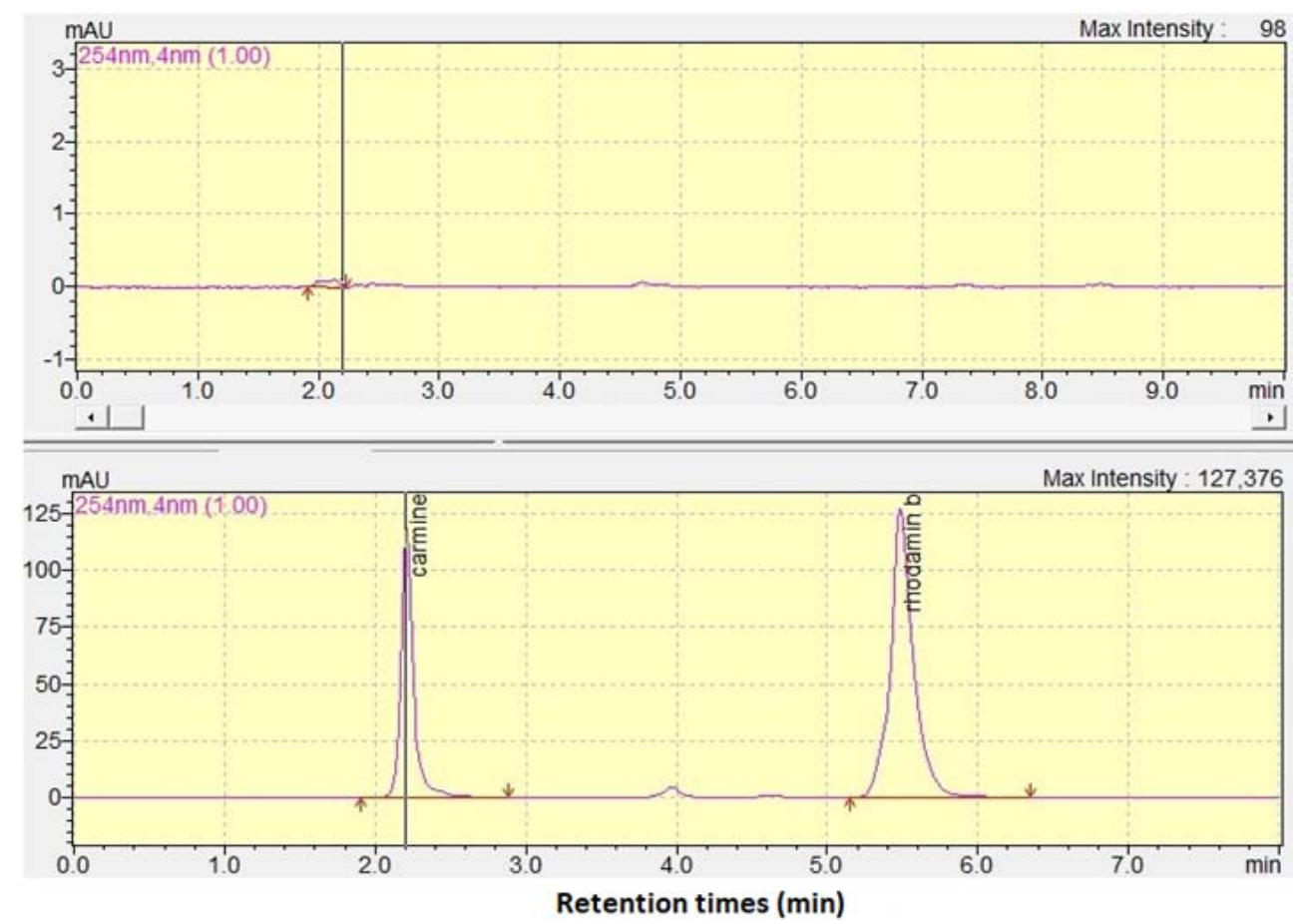

Fig. 5: The chromatogram of lipstick samples above and lipstick samples spiked with standards of carmine and Rhodamine Below

\section{CONCLUSION}

RP-HPLC using C18 column with PDA detector has been successfully validated for quantitative analysis of CAR and RHO B in lipstick samples. The developed method was accurate and precise as indicated by acceptable values of relative standard deviation (RSD) and recovery percentage. The validated RP-HPLC method was simple with no excessive sample preparation and suitable to be used for routine analysis of dyes (CAR and RHO B) in lipstick samples.

\section{ACKNOWLEDGMENT}

The author acknowledges to Indonesian National Agency of Drug and Food Control, Indonesian National Agency of Drug and Food
Control in Surabaya, East Java, and Indonesian National Agency of Drug and Food Control in Yogyakarta for financial support and instrument facilities.

\section{CONFLICT OF INTERESTS}

The authors have declared "no conflicts of interest with respect to the research, authorship, and/or publication of this article".

\section{AUTHORS CONTRIBUTIONS}

RN performed research activity, compiled data, and prepared manuscript. AR and SM designed research activities, prepared manuscript and made critical thinking on the manuscript. 


\section{REFERENCES}

1. Nourmoradi H, Foroghi M, Farhadkhani M, Vahid Dastjerdi M. Assessment of lead and cadmium levels in frequently used cosmetic products in Iran.J Environ Public Health 2013;2013:962-727.

2. Wang J, Kay AB, Fletcher J, Formica MK, McAlindon TE. Is lipstick associated with the development of systemic lupus erythematosus (SLE)? Clin Rheumatol 2008;27:1183-7.

3. Kanekar H, Khale A. Coloring agents: current regulatory perspective for coloring agents intended for pharmaceutical and cosmetic use. Int J Pharm Phytopharmacol Res 2014;2:1-20.

4. Sabnis RW. Handbook of Biological Dyes and Stains: Synthesis and Industrial Applications, John Wiley and Sons, Inc., Hoboken, NJ, USA; 2010. p. 56.

5. Tatebe C, Zhong X, Ohtsuki T, Kubota H, Sato K, Akiyama H. A simple and rapid chromatographic method to determine unauthorized basic colorants (rhodamine B, auramine 0 , and pararosaniline) in processed foods. Food Sci Nutr 2014;2:547-56.

6. Greenhawt MJ, Baldwin JL. Carmine dye and cochineal extract: hidden allergens no more. Annals Allergy Asthma Immun 2009;103:73-5.

7. Fisher P. Review of using rhodamine $b$ as a marker for wildlife studies. Wildlife Soc Bull 1999;27:318-29.

8. Rastogi SC, Barwick VI, Carter SV. Identification of organic colourants in cosmetics by HPLC-diode array detection. Chromatographia 1997;45:215-28.

9. Miranda Bermudez E, Harp BP, Barrows JN. Qualitative Identification of permitted and non-permitted color additives in cosmetics. J AOAC Int 2014;97:1039-47.
10. Feng F, Zhao Y, Yong W, Sun L, Jiang G, Chu X. Highly sensitive and accurate screening of 40 dyes in soft drinks by liquid chromatography-electrospray tandem mass spectrometry. J Chromatogr B 2011;879:1813-8.

11. Liu X, Yu D, Yu Y, Ji S. Preparation of a magnetic molecularly imprinted polymer for selective recognition of rhodamine B. Appl Surface Sci 2014;320:138-45.

12. Gładysz M, Krol M, Koscielniak P. Differentiation of red lipsticks using the attenuated total reflection technique supported by two chemometric methods. Forensic Sci Int 2017;280:130-8.

13. Sharma S, Goyal S, Chauhan K. A review on analytical method development and validation. Int J Appl Pharm 2018;10:8-15.

14. Guidelines for validation of Analytical Procedures, Q2 (R1), ICH; 2005.

15. Kuber RB. Method development and validation for estimation of curcumin and piperine by RP-HPLC. Int J Appl Pharm 2019;11:216-21.

16. Cruz DD, Babu A, Joshy E, Anesh TP. Bioanalytical method development and validation of ticagrelor by RP-HPLC. Int J Appl Pharm 2017;9:51-4.

17. Miller JN, Miller JC. Statistics and chemometrics for analytical chemistry, Prentice Hall, England; 2010.

18. Gonzalez AG, Herrador MA. A practical guide to analytical method validation, including measurement uncertainty and accuracy profiles. Trends Anal Chem 2007;26:227-38.

19. Noviana E, Pranowo HD, Astuti A, Rohman A. Validation of mercury analyzer for determination of mercury in snake fruit. Int Food Res J 2012;18:933-6. 